\title{
"Soft" system of coordinates in regular simplexes
}

\section{Tomas Georgievich Petrov}

St. Petersburg State University, Institute of Earth Science, St. Petersburg, Russia

\section{Email address:}

tomas_petrov@rambler.ru, tgpnever@gmail.com

\section{To cite this article:}

Tomas Georgievich Petrov. "Soft” System of Coordinates in Regular Simplexes. International Journal of Intelligent Information Systems. Vol. 4, No. 1, 2015, pp. 1-7. doi: 10.11648/j.ijiis.20150401.11

\begin{abstract}
In different areas of knowledge, there are common problems of coding and ordering of multicomponent compositions of objects as well as representation of their processes of change. These problems are solved by the languagemethod RHAT. It can be considered as a coordinate system of regions in space limited by regular simplex. Here R is a sequence of composition components by decrease - names of sectors in the simplex distinguished by hypermedians, a semiquantitative substantial characteristic of compositions, "word" of a new type; $\mathrm{H}$ - Shannon information entropy - entropy of mixing; A - anentropy - entropy of separation; $\mathrm{T}$ - tolerance. The arrangement of coordinates allows obtaining an alphabetical hierarchical periodic system of compositions (HPSC) of objects of different nature, in particular, the Hierarchical Periodic System of Chemical Compositions (HPSCC) that uses the Periodic System of Elements (PSE) as an alphabet, as well as the Hierarchical Periodic System of Molecular Compositions (HPSMC) that uses HPSCC as an alphabet. Diagrams HA or HT are designed to display random and ordered sets of compositions of any nature as the most appropriate means of studying the processes of separation and mixing. The applicability of the method has been tested on analytical materials in natural and social science fields.
\end{abstract}

Keywords: Regular Simplex, Coordinate System of Region, Information Entropy, Entropy of Separation, Anentropy, Ultrapurification, Tolerance, Information Language, Hypermedian, Hierarchical Periodic System

\section{Introduction}

Background of the method, common for the natural sciences, is presented in $[3,9,10]$. Mostly it relates to the multicomponent nature of objects (cenoses, communities, mixtures), namely: uncertainty of boundaries between compositions of such objects, which is significant in nature and in all areas of human activity, including dispensing medicines and food, choice of winner, study of isomorphism in crystals, financial capacity determination, military solutions, etc.) and uncertainty of naming what is inside the boundaries, difficulties in visibility and ordering of object compositions, diversity of content measurement units and disproportionality of large and small in the same composition, difficulty in identifying the general direction of the evolution of compositions under multi-directional changes in individual components. To reduce the complexity of solving these problems, information language method, or rankentropy method (hereinafter - way or method) for displaying, particularly, coding of compositions RHAT has been proposed. Here $R$ is a sequence of symbols of composition components by reduction of their contents; $H$ is $\mathrm{C}$. Shannon information entropy as entropy of mixing; A - anentropy [6$13,15,20,21$,$] , as entropy of separation [13,15] ; \mathrm{T}-$ tolerance $[10,15]$ - as "entropy of purification". The method has been repeatedly described in $[69-13 ; 17]$, and will be outlined briefly here. Details are presented in [21].

Method wasinitially proposed particularly for convolution of chemical compositions of rocks and minerals, but later its usefulness has been shown in solving conceptual tasks in various fields of knowledge [4, 13, 15,16, 19, 25; 26].

Method is usually set out in terms of algebra, information theory. Here, we present it at the elementary level as a way of describing - coordination - coding of an area in space limited by a regular simplex with equal distances from the center to all vertices, and distances between all vertices. Of all simplexes, triangle has long been a "sacred cow" of mineralogists, petrologists, and many other geologists, at the same time becoming a dead end in the representation of multicomponent systems on paper. Background demand of visibility at preservation of the traditional approach has not enabled to pass into the volume and work in it, since even imaging of tetrahedron content is problematic. Representation of 5-10-50-component analysis without a 
radical convolution of analysis is impossible, and, at the same time, chemical analyses with 50 or more elements have become quite common. On the other hand, actively working "naturalists" and "humanitarians" accumulate thousands of chemical, mineral, biospecies, age, linguistic "analyses". There are problems in putting them in order, discovering duplicates, defects, seeing the material as a whole (rather than its individual components, or the relations between two or three of them), seeing the relationship between compositions, concentrations and rarefactions, banality and originality of individual analyses, identifying the direction of change and path length of the evolution of composition, and, amid all this, in detecting a law or prohibition of a certain already formed hypothesis: all that was worth of the efforts spent on the method development and is worth of the work to become acquainted with it.

The method can be considered both as a way of coding, and as a "soft" system of coordinates, meaning by its "softness" lack of an exact solution of the inverse problem with the number of variables exceeding the number of equations. Thus, 40-element composition analysis of a complex mineral, tourmaline, contains 50 unknowns convolved in 4 equations: $H, A, T$ and $\operatorname{Sum} p_{\mathrm{i}}=1$.

Under "soft" coordinate system we mean description of area position (and not point, as in a "hard" one of some space, in this case, space of a regular simplex).

Purpose of the article is to pay attention to the universal encoding method of multicomponent compositions $R H A T$, as well as to the problems in mathematics arising from the use of the method in different fields of knowledge.

\section{2. $R, H, A, T$ as Coordinates in a Simplex Space}

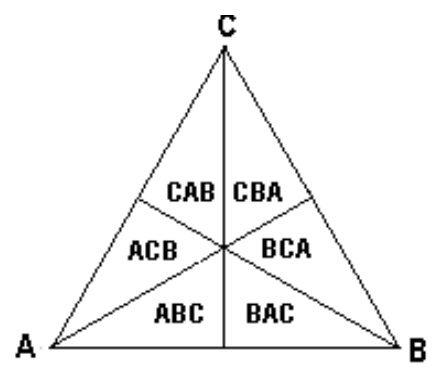

Figure 1. Simplex with names of sectors distinguished by medians

First coordinate: $R$, rank formula of composition, rating, is a sequence of symbols (or names) of $n$ components (selected from the total possible in this branch of knowledge number $N$ ) by reduction of their contents $p$, when $\operatorname{Sum} p_{\mathrm{i}}=1$. This series (e.g. "quartz-feldspar-mica", a sequence by reduction of contents of principal rock minerals - granite) is a sector name in a regular simplex having three vertices when split by medians in a triangle (Fig. 1). Rating $R$, a semiquantitative while substantial characteristic of composition, is regarded as the major semantic part of the composition convolution in the form of RHAT code. Four-component composition requires a tetrahedron when split by median planes, and further - an unthinkable regular polyhedron with median hyperplanes. In the simplex vertices all $p=1$, that is, here are absolutely pure components (not present in nature, but more on that below). In the simplex center $p_{1}=p_{2}=p_{3}==\ldots p_{\mathrm{n}}$ $=1 / n$. In volume of a simplex with $n$ vertices, the number of sectors $K=n$ ! An elementary example of sector naming is shown in Fig. 1.

Contents of the first component are in the range $1 / n-1$. In other ranks, $0-1 / n$.

Besides the said role, $R$ is a means of component selection if the sample contains analyses of different lengths, which is critical for subsequent calculations of composition characteristics. Namely, for comparability of the processing results of analyses with varying $n$ detail, after ranking of the initial data, further computing includes the amount of components not greater than $n$ of the shortest analysis. This does not eliminate the possibility to find in the analysis a component, the content of which is smaller than would be in the case of a more complete analysis. Therefore, there is a choice: increasing of details and reduction of confidence in the correctness of the result, or increasing of confidence at reduction of analysis details.

\subsection{Information Entropy $\mathrm{H}$ or En}

The second coordinate: $H$, Shannon information entropy up to a constant is an analog of the thermodynamic entropy of mixing [2] and the measure of complexity, diversity of system composition. (The diversity problem is discussed in [26]). Evaluation by formula $H=-\operatorname{Sum} p_{\mathrm{i}} \ln p_{\mathrm{i}}$. To reduce to the interval $0-1$ we use $E n=H / \ln n$, where $\ln n=H \max$. Maximum value of contribution of one component in entropy $-p_{\mathrm{i}} \ln p_{\mathrm{i}}$ for logarithms with any base is at $p=0.368 \ldots$ In a simplex-triangleisolines of entropy normalized to 1 are shown in Fig. 4. In accordance with isoline appearance, entropy can be regarded as a generalized distance from simplex vertices to the composition point.

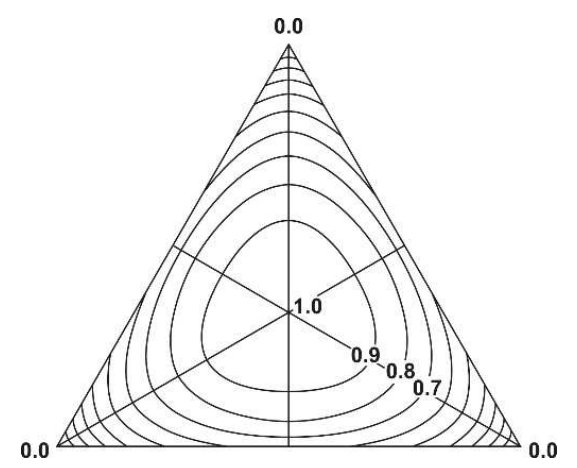

Figure 2. Isolines of entropy normalized to the interval 041 .

\subsection{Anentropy $A$ or $A n$}

The third coordinate, anentropy [6, p.34; 9, p.17] is regarded as a measure of smallness of small components and calculated by formula $A=-1 / n^{*} \operatorname{Sum} \ln p_{\mathrm{i}}-\ln n$. According to the formula, $A$ exists in the range $0-+\infty$. To reduce to the 
interval 0-1, convenient for many applications diagrammaticform (in combination with entropy), $A$ of a real composition is divided by anentropy of a "simple analytically perfectly clean" system[9, p.18]. This is necessary, since zero in composition responds to the logarithm of minus infinity. For maximum pure composition is taken composition, in which $p_{1}=1-(n-1)^{*}, p_{2}=p_{3}=\ldots=p_{\mathrm{n}}=$, where are contents equal to half sensitivity of the analysis method. This trick is dictated by V.I. Vernadsky "ubiquity principle" (as it is called, slightly reinforcing the position of its author) [1. p.518-527], according to which any macroobject contains all elements of the Periodic System. Let us add: tricks are useful as well (with their ) when considering compositions of any real systems, whether mineral, national, age etc., in which the smallest contents are almost always underestimated - to the detriment of the meaning of quantitative estimates.

Anentropy isolines in a regular triangle are shown in Fig. 5. In accordance with their nature, anentropy can be considered a generalized distance from the simplex center to the composition point.

Anentropy is regarded as entropy of separation, since many observations of trajectories of processes of separation, for example, by grain size in sedimentary rocks, population age, during mineral processing, show its increase [13, p.24; 22].

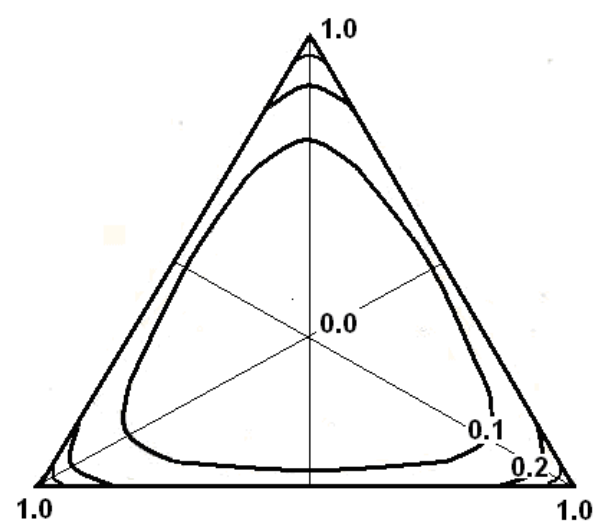

Figure 3. Isolines of anentropy normalized to the interval 0-1.

\subsection{Tolerance $-T$}

Tolerance as a coordinate $[10$, p.30; 13, p.24], the contribution to which is $1 / p$, is calculated by formula $T=$ $\ln \left[1 / n * \operatorname{Sum}\left(1 / p_{\mathrm{i}}\right)\right]-\ln n$. We do not show isolines of normalized tolerance because they are pressed to the simplex vertices even in a greater extent than anentropy isolines. This characteristic is most applicable for estimating the degree of composition closeness to the simplex vertex, to a state of perfect purity of composition.

To distinguish it from the entropy of separation (as a means to display widely used methods of relatively coarse separation), tolerance, the means to show processes of extremely pure substances, can be called entropy of purification.

From the viewpoint of information theory, the value of tolerance is the logarithm of average signal waiting time at their uniform arrival.

Contributions of three quantitativecomposition coordinates are shown in Fig. 6.

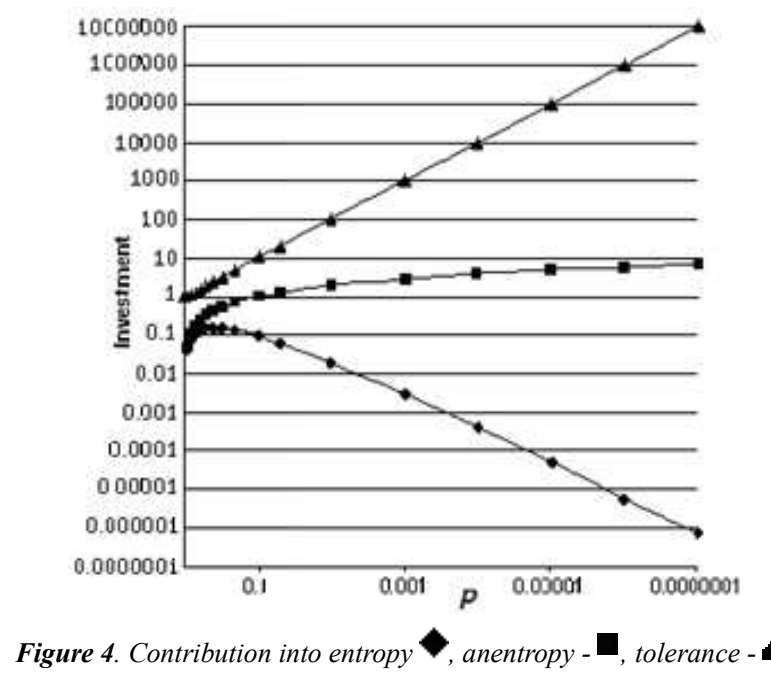

Contributions into $A$ and $T$ are functionally dependent on the contribution into entropy. If we take the contribution into entropy $(-p \ln p)$ for a primitive function, its first derivative with respect to $p$ minus one will be contribution into anentropy $(-\ln p)$, contribution into tolerance - the second derivative with respect to contribution into entropy and the first one with respect to contribution into anentropy $(1 / p)$. Thus, both anentropy and tolerance are natural outcomes, subsequent development stages of the ideology embedded in C. Shannon information entropy. The transition from the widely applicable one-dimensional data representation of the complexity of systems to the two-dimensional, diagrammatic and three-dimensional, spatial, that is to the means more filled (or richer) in content is implemented this way.

\section{Systematics - Ordering of Composition Codes}

RHAT composition coordinates (with descriptions of specific objects) are recorded in a row and arranged in a column, as shown in Table 1. For this a set, series of RHAT coordinates is taken as a word that uses three alphabets. One is letter, for rank formulas playing the role of "roots", semantic meaning of a word, and two numerical: for entropy ("suffix") -by reduction of $H$ values and for $A$ and $T$ - by their increase ("inflexions"). Ordering is done according to the known linguistic principle, consistently. First roots are ordered, then suffixes, and further inflexions.

In general, it is desirable to use any "intensional" alphabets as symbols in rank formula $R[25,28]$. They are understood as alphabets which have semantic links between adjacent symbols (entities), as opposed to "natural" alphabets, in which such links either do not exist or are very weak [28]. It is known that dictionaries using "natural" alphabets usually put close words and form groups having similarity in inscription (alt, altar, alter, altimeter, altruism...) and rare 
with a similar sense. Unlike the latter, when using the most famous intensionalalphabet in encoding chemical compositions of geological objects, the Periodic Table of Elements, groups of rank formulas OSiAlH..OSiAlNa... OSiAlK contain different, but related in chemical composition objects. They have a common name "alumosilicates"; some of them are presented in Tab. 1. When rank formula length increases, certainty of allocating a portion of space, to which the composition belongs, and, on the other hand, the degree of generalization at its reduction are growing.

In case of equal $R$, ordering of composition coordinates is made by reduction of $H$, the preferential direction of entropy change during separation [29]. (Separation is understood as formation of two or more systems from one that differ in composition. Mixing is the opposite process of forming a relatively homogeneous system of two or more differing in composition). In case of equal $H$, ordering of coordinate lines is made in the usually opposite direction of $A$ and $T$ changes, i.e. by their increase.

Such ordering results in a Hierarchical Linear Periodic System of Compositions, in particular, chemical, when coordinates of chemical compositions of objects are ordered [12]. Table repeated from [12] shows a sample of code coordinates from the existing Database of Chemical Compositions that contains more than 80000 entries. This is a negligible part of a full table (probably impossible to construct), given the number of stable elements (88?) and taking into account $\boldsymbol{n}=10$ elements in $R$. Horizontal lines in the table are dividers revealing the hierarchical structure of the system. Equal signs mean that $p_{\mathrm{n}} / p_{\mathrm{n}+1} \leq 1.15$, i.e. indicate the proximity of contents of the adjacent components in a rank formula.

Table 1. Sample from the "Chemical Compositions of the Universe" Database

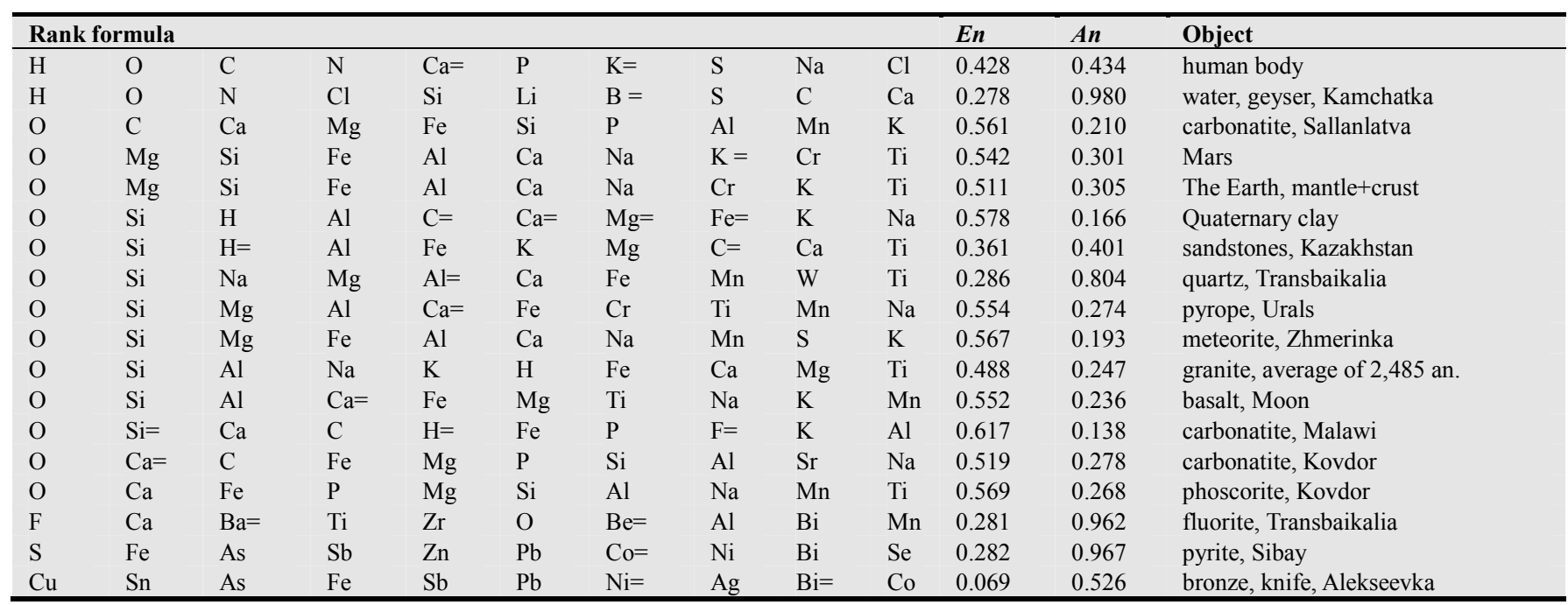

The database of object descriptions contains a reference to the source, the number of table and analysis in it, as well as other specific information about the object.

\section{Properties of Databases Systematized Using RHAT Method}

Database, a sample of which is shown in Table, has the following properties:

Versatility - with respect to the possible diversity of chemical compositions (hence the database name), since there is no chemical (let us add: and any other) composition, which would be impossible to imagine in $R H A(T)$ form;

Linearity - has no branching and therefore is extremely simple;

Algorithmicity - when constructing;

Openness - to expand the list of components;

Stability, i.e., the system allows for the removal and inclusion of new records without changing the order of the others;

Countability - the maximum number of rank formulas
$U$ (from Universum) can be defined as the number of permutations without repetitions of $N$ possible components of this alphabet by $n$, i.e. $U=N ! /(N-n)$ !. For $N=88$ and $n=10$ we have $U=1.64 \mathrm{E}+19$. The chemical Universe can be placed in this catalog.

Completeness - for a certain alphabet length and a certain length of rank formula there are no and cannot be other rank formulas over the number of permutations, as defined above. Therefore, for these $N$ and $n$ the sequence is a Universum of existing, possible and impossible compositions in this field of knowledge;

Hierarchicity- ordering, as in alphabetical dictionaries, is primarily done by the first, the highest rank (first letter). Further, inside the rank formulas with the same first rank ordering takes place by the second, lower rank (second letter), etc. As a result, we have a hierarchical ordering of codes.Rank formula with length $\boldsymbol{n}$ is an ordered list of all higher taxa;

Periodicity - rank formulas of objects with similar composition are arranged in table in groups, between which there are other, much different ones; thus, in Table 1 rank 
formulas OCCa...OCaC... located at opposite ends of the table belong to rocks of the same facies - these are carbonate varieties.

Arrangement of objects in a system manifests connection with the procedure of changing some properties of object ordering in the original alphabet (here, in the Periodic Table). Thus, for chemical compositions, from the classification beginning to its end, average atomic masses of objects, their densities grow statistically, occurrence in nature decreases statistically.

\section{Entropy diagrams $H A$ and $H T$}

Values of all three numeric coordinates $H, A, T$ depend on the number of components. Therefore, if desired to deal with full analyses, very often having different $n$, their comparison will be either difficult, or impossible, or this fact can be used (but at a sufficiently large amount of data) to identify the structure of the information space. Specifically, to image variety of materials by their detail (analyses length), that is clearly manifested in $H A$ diagram of age distributions of population in countries of the world [14].

Bearing in mind great importance of the issue let us partially repeat. For comparability of data, $R$ lengths are standardized on some general level and, after renormalization of sums of truncated analyses to the unity, HAT is calculated. To account for the fullest possible information, standard should be brought into proximity with the length of the shortest analysis in the sample. It should be borne in mind that the shorter the analysis, the greater the chance to lose underestimated components and include in the calculation lower values than would have been at a more complete analysis, respectively, the higher anentropy can be [21, p.68]. There is reason to believe that the delay in the use of entropy in some branches of knowledge, particularly in geology, is associated with ignoring the need to standardize the analysis details before computations.

Field of allowable EnAn values for a 10-component system is shown in Fig. 5. The upper limit was obtained by mixing of compositions described in detail in [9, p.17]. Figures near the points along the upper curve correspond to the number of components with equal contents, assuming that the remaining ones (10 minus "figure"), supplementing the analysis to ten components, had contents of half sensitivity of the analysis method taken for 0.00005 . (This value corresponds approximately to the sensitivity of "wet" analyses that have been ubiquitous until recently and did not lose value with the advent of new technologies). The lower curve meets the condition: $p_{1}=1-\left(p_{2}+p_{3}+\ldots+p_{10}\right)$, thereby: $p_{2}=p_{3}=p_{4}=\ldots=p_{10}$.

Abbreviations $\mathrm{S}$ denote point of original compositions (Start), F (Final) - final product compositions. The diagram shows the typical directions of composition change during separation (two S-F patterns on the left) and mixing processes (two patterns on the right), found in tracing the evolution trajectories of compositions in dozens of real processes of change in composition of rocks and minerals.
Featured direction ratios during mixing - combination of two different compositions into one, and during separation formation of two or more different compositions from one illustrate Yu.V. Shurubor's theorems of preferred directions of entropy change during mixing and separation [29].

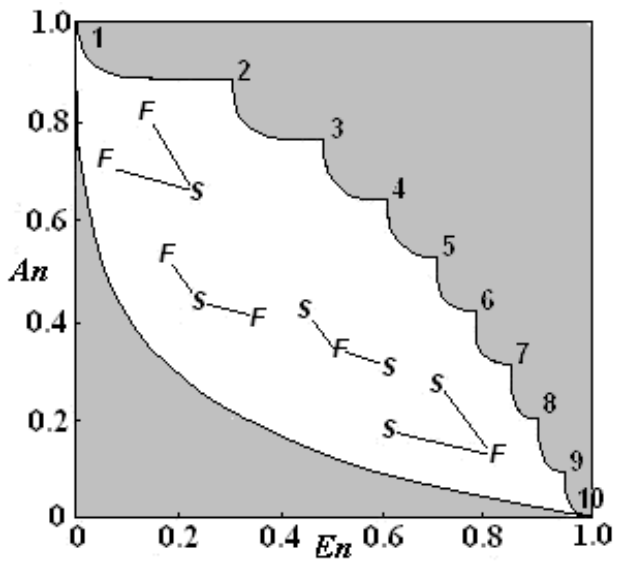

Figure 5. Limits of EnAn field and typical directions of composition evolution during separation and mixing (explanation in the text).

Separation and mixing processes shownin Figure 5, "pull" points of compositions to vertex No.1, if content of one component is growing in composition (native elements, or close to mono-ethnic community); to No.2, if, except for two components with equal contents, the remaining components are removed (purification of rock salt $\mathrm{NaCl}$ ); to No.3, if a three-element compound of $\mathrm{KOH}, \mathrm{NaOH}$ type is purified, and so on. Reverse movement, towards the simplex center (No. 10 in the diagram) corresponds to mixing processes. Neither simplex center nor its vertices contain real (or at least chemical) compositions, since to achieve these points, nonexistent in nature and in laboratory ideal pure substances are required. Everything happens between these two extremes in a cyclic implementation of separations and mixings. For example, in geology, formation of granite is separation, then hydration of certain minerals, mixing, further disintegration of rock with removal of having become mobile solutions and clays - separation, entering of granite degradation products in streams and soil-mixing and so on, for each preserved chemical element of the primarysystem, an eternal change of participation in mixing and separation processes. In biocenoses, birth and death of organisms is imposed on separation-mixing processes, but the reciprocating motion of a point noting the seasonal cycles in $H A$ diagram will occur monotonically as long as the ecosystem is not "sick" or starts to disintegrate.

According to the data published in [5] and calculations conducted by the author, it is known that the scatter in EnAn data in the middle ofthe diagram when using standard chemical analysis in geology for $n=10$ is approximately \pm 0.005 in both axes.

Description of the diagram ispresented in $[9,13]$ andin most detailin [21]. Model of the separation-mixing process in qualitative presentation is given in[15]. 


\section{Unresolved Problems}

Development of the method has mainly taken place "broadwise", towards the testing of opportunities and meaningfulness of results when using the method in various areas of knowledge, which are already about 20. At the same time, the mathematical aspect has been apparently developed insufficiently.

Exceeding the number of unknowns on the number of equations implies uncertainty when solving the inverse problem, inability to determine $p_{\mathrm{i}}$ for $H A$ or $H T$ data. However, in each triple combination: $R_{\mathrm{i}}, H, A$, (or $R_{\mathrm{i}}, H, T$ ), $p_{\mathrm{i}}$ range is not unlimited. There is a task to find a way for calculating $p_{\min }$ and $p_{\max }$ for each $R_{\mathrm{i}}$ of the system with cardinal $n$ for the set HA,HT, EnAn at $p_{\min }=$. In other words, what are shapes of "bodies of $R_{\mathrm{i}}$ contents" in projections on the fields of EnAn, HA, and HT diagrams?

Another question is very important in the practice of studying evolution of compositions. All mathematically made mixings (interpolations) of compositions, that is when signs of change are preserved, and the vast majority of the examined processes of evolution of compositions of natural objects give arcs convex downward or downward - to the right (such as given in [13] in Fig. 7). [3] describes the process, which has a curve of the opposite form: bulge upward - to the left. As has been well established, it took place in two stages: the first one, with the normal trend of separation - decrease of $H$, growth of $A$, and the second one with the opposite trend, mixing. There was a task to determine whether this type of curve may correspond to a "one-way" ("monotonic") process, that is, without changing the signs of change in content? If we prove that this is impossible, there will be confidence that the processes of this type are not monotonic.

\section{Some Results and Prospects}

RHAT is a universal method for describing compositions, one of the methods along with the principal component analysis, cluster analysis, and others that may be included in generalizing discipline "Composistics" enabling to work with compositions, intensities, weights, values of components of any complex systems [ 8 p.271]. The method provides a wide range of possibilities; alternatives for applying it are described in [11]. The same publication lists three types of work, namely: with primarily disordered analytical materials, with a database, and with materials ordered in space and time.

Proposed in 1971, the method can be said to lie long, it was not properly claimed in geology due to several reasons, among them probably the most important: a) unconventional measurement units - atomic fractions accepted by the author for a universal approach - for chemical composition (instead of remaining since the 19th century oxides such as $\mathrm{SiO} 2$, $\mathrm{Al} 2 \mathrm{O} 3, \mathrm{FeO} \ldots$ ); b) integrated approach to complex compositions at prevailing in diagrams consideration of only 2-3 components of composition, c) more than once mentioned logarithmic curve. It is also very likely: $d$ ) distrust of a geologist-crystallographer who encroached on the solution of problems that appeared to be insoluble in the 70ies: uniform ordering of all chemical and mineral compositions, and placing in a single diagram, having a genetic interpretation, compositions of objects of any nature, and thus being able to display and study the evolution of the whole world of multicomponent compositions in diagrams, and not using one-dimensional sequences of information entropy. The proposed method for construction of a twoparameter alphabet was the first step to address the issue of content encoding of crystal-chemical structural formulas of minerals [16] (and not only them), in combination with the $R$-dictionary-catalog of chemical compositions of minerals [18]; it offered an opportunity to create a unified structuralchemical classification of real minerals and to develop on its basis the looming "RHAT dictionary-catalog of crystalchemical compositions of minerals".

Work on the method and several others is supported by original Petros 3 software enabling to deal with 100 alphabets, to form databases, including bibliographic one, to perform calculations of correlation coefficients and distances of several kinds, convolutions of large amounts of information in the form of "generalized rank formulas" etc. [10].

\section{Conclusion}

The author will consider his mission accomplished, if he draws attention to the fields of mathematics, that enable a consistent encoding of compositions of objects of any nature and development of an algorithm for constructing rankentropy alphabetically ordered Hierarchical Periodic Systems of Compositions, in contrast to the original Periodic System of Elements, in addition, showing the processes of composition change, revealing common in disparate at first glance processes in stagnating, living and social, in particular, regarding them as separation and mixing - implementation of differentiation and integration, recognized fundamental by Herbert Spencer.

\section{Acknowledgements}

The author thanks his long-time collaborator N.I. Krasnova for dedicated efforts to disseminate the method, S.V. Moshkin for programming and implementation of the endless requests to add another method in the software, to explain how-to, A.G. Bulakh, Yu.L. Voytekhovsky, and recently deceased V.V. Gordienko for deep understanding and high public estimation of the method, S.V. Chebanov for continued support and strengthening methodological fundamentals of the method, A.A. Andriyanets-Buyko, V.A. Glebovitsky, V.Ya. Vasiliev, V.N. Dech, I.S. Sedova, P.B. Sokolov, O.I. Farafonova, V.V. Khaustov, A.V. Shuisky who contributed to the author in his work towards formation and development of the method, and all others who resisted the method development in different ways, prompting the author to a more thorough understanding and description of his work. Special thanks to O.Yu. Bogdanova, whose translation 
of the papers promoted their publication in the multidisciplinary scientific environment.

\section{References}

[1] Vernadsky V.I. On the scattering of chemical elements. Selected works. V.1. AS USSR Press. 1954. - Pp. 517-527

[2] Moelwyn-Hughes E.A. Physical chemistry. Mon. In 2 v. M. IIL, $1962-1148$ p.

[3] Gordienko V.V., Petrov T.G. Studying of rare-metal pegmatites using RHA language. // RMS Proceedings. - Part 110. - Iss.5 - 1981 Pp. 546-558

[4] Kramarenko S.S. Features of using entropy information analysis for quantitative criteria of biological objects // Bull. Samara Sci. Centre of RAS. - 2005

[5] Kukharenko A.A., Ilyinsky G.A., Ivanova T.N. et al. Clarkes of the Khibiny alkaline massif // RMS Proceedings 1968. Part 97. Iss. 2. - Pp.133-149

[6] Petrov T.G. Substantiation of a version of general classification of geochemical systems. // LSU Bulletin. No. 18 - 1971. - Pp. 30-38.

[7] Petrov T.G. A problem of separation and mixing in inorganic systems. In: Geology. Ed by V.T. Trofimov, v.2. - Moscow State University. - 1995. - Pp.181-186.

[8] Petrov T.G. Language-method RHA for describing, systematizing, and studying changes in compositions of any nature. // In: Cenological studies. Ed. B.I. Kudrin. Iss. 1 Mathematical description of cenoses and technetical laws. Abakan: SRC - 1996. - Pp.256-273

[9] Petrov T.G. Information language for description of multicomponent objects composition // Scientific and technical information. Ser. 2. - 2001. - No. 3. - Pp. 8-18.

[10] Petrov T.G. Rank-entropy approach to description of compositions of geological objects and their changes (exemplified by geological cenology). // General and applied cenology. 2007. - No.5. - Pp.27-33

[11] Petrov T.G. RHA method as a solution of systematization problem of analytical data on the material composition of geological objects. Otechestvennaja geology. 2008. - No.4. Pp.98-105.

[12] Petrov T.G. Hierarchical periodical system of chemical compositions and its association with the periodical system of elements. // SPbSU Bulletin. Ser. 7. 2009. - Iss. 2. Pp. 21-28.

[13] Petrov T.G. Graphic Representation of Evolutionary Processes of Compositions of Multicomponent Objects of Any Nature // Automatic Documentation and Mathematical Linguistics. 2012. - V. 46. - No. 2. - pp. 79-93. (C) Allerton Press, Inc., DOI: $10.3103 / \mathrm{S} 0005105512020045$

[14] Petrov T.G. RHA method for coding, systematizing, and displaying the changes in age compositions of population. // Coll. dedicated to the 60th anniversary of S.V. Chebanov (in press)
[15] PetrovT.G. Separation-Mixing as a Model of Composition Evolution of any Nature. // J. Systemics, Cybernetics and Informatics V. 12. - N 1 - 2014. - Pp. 76-81.

[16] Petrov T.G., Andriyanets-Buyko A.A., Moshkin S.V. A TwoParameter Alphabet for Coding Structural-Chemical Information and its Systematization (Using the Example of Tourmaline).// Automatic Documentation and Mathematical Linguistics. 2012. - V. 46 -.No. 1. - pp. 40-49. SpringerLink Online- DOI 10.3103/S0005105512010086

[17] PetrovT.G., Moshkin Sergey V. RHA(T)-System for Coding of Discrete Distributions and Their Alteration Processes. // Proc. The 3rd International Multi-Conference on Complexity, Informatics and Cybernetics IMCIC 2012. - 2012. - Pp. 1216.

[18] Petrov T.G., Krasnova N.I. R-dictionary-catalogue of mineral chemical compositions. Mon. St. Petersburg, - "Nauka". 2010. -150 p.

[19] Petrov T.G., Krasnova N.I. RHA-coding of mineral composition of crystalline rocks and method of their systematization. // Modern problems of magmatism and metamorphism. Mon. Vol.2 - St. Petersburg - 2012 - Pp. 120123

[20] Petrov T.G., Moshkin S.V. RHA method and its implementation in Petros-3 software system. Calculations in geology. 2011. - No.1. - Pp. 50-53.

[21] Petrov T.G., Farafonova O.I. Information-component analysis. RHA method. Study guide. St. Petersburg - 2005. - 168 p.

[22] Petrov T.G., Farafonova O.I., Sokolov P.B. Informationalentropic characteristics of composition of minerals and rocks as a reflection of crystallization process stress: RMS Proceedings - 2003. - No.2. - Pp. 33-40.

[23] Khaustov V.V. Role of deep geodynamics in hydrolithosphere formation (exemplified by the Caspian-Caucasus segment of the Alpine-Himalayan mobile belt. Abstract of Doc. Thes. St. Petersburg $-2011-40 \mathrm{p}$.

[24] Khaustov V.V. Towards the problem of mud volcano water formation in the South Caspian basin // "Space and Time" Almanac. T. 1. - Iss. 1 - 2012.

[25] Chebanov S.V. Logical fundamentals of linguistic typology. Mon. Vilnius. VLANI. 1996. - 92 p.

[26] Chebanov S.V. Optimality and extremality in culture, zipfiades and Lotman's law. // In: Technogenic selforganization and mathematical apparatus of cenologic studies. M SRC. - Iss. 28. - 2005 - Pp.411-428

[27] Chebanov S.V. Dynamics of cultural forms.// In: Fundamental problems of cultural studies in 4 vol. Cultural dynamics v.3. St. Petersburg. Aletheia. 2008. - Pp. 184-197

[28] Chebanov S.V., Petrov T.G. Intensionality, intensional alphabets, intensional words and dictionaries // In: Actual problems of modern cognitive science. Ivanovo. - 2013. Pp.239-266

[29] ShuruborYu.V. On one property of complexity measure of geochemical systems.// Proceedings of the AS USSR. - 1972. - V. 205. - No.2. - Pp. 453-456 